\title{
Article \\ Optimization of Computed Tomography Angiography Protocols for Follow-Up Type B Aortic Dissection Patients by Using 3D Printed Model
}

\author{
Chia-An Wu ${ }^{1}$, Andrew Squelch ${ }^{2}$, , Shirley Jansen ${ }^{3,4,5,6}$ and Zhonghua Sun $1,7, * \mathbb{C}$ \\ 1 Discipline of Medical Radiation Science, Curtin Medical School, Curtin University, Perth 6845, Australia; \\ chia-an.wu@postgrad.curtin.edu.au \\ 2 Faculty of Science and Engineering, WA School of Mines: Minerals, Energy and Chemical Engineering, \\ Curtin University, Perth 6845, Australia; A.Squelch@curtin.edu.au \\ 3 Department of Vascular and Endovascular Surgery, Sir Charles Gairdner Hospital, Perth 6009, Australia; \\ Shirley.Jansen@health.wa.gov.au \\ 4 Curtin Medical School, Curtin University, Perth 6845, Australia \\ 5 Faculty of Health and Medical Sciences, University of Western Australia, Crawley 6009, Australia \\ 6 Heart and Vascular Research Institute, Harry Perkins Institute for Medical Research, Perth 6009, Australia \\ 7 Curtin Health Innovation Research Institute, Curtin University, Perth 6845, Australia \\ * Correspondence: z.sun@curtin.edu.au; Tel.: +61-8-9266-7509; Fax: +61-8-9266-2377
}

Citation: Wu, C.-A.; Squelch, A.; Jansen, S.; Sun, Z. Optimization of Computed Tomography

Angiography Protocols for Follow-Up Type B Aortic Dissection Patients by Using 3D Printed Model. Appl. Sci. 2021, 11, 6844. https://doi.org/ 10.3390/app11156844

Academic Editor: Flavia Ravelli

Received: 20 May 2021

Accepted: 23 July 2021

Published: 25 July 2021

Publisher's Note: MDPI stays neutral with regard to jurisdictional claims in published maps and institutional affiliations.

Copyright: () 2021 by the authors. Licensee MDPI, Basel, Switzerland. This article is an open access article distributed under the terms and conditions of the Creative Commons Attribution (CC BY) license (https:// creativecommons.org/licenses/by/ $4.0 /)$.

\begin{abstract}
Thoracic endovascular aortic repair (TEVAR) is a life-saving therapy for type B aortic dissection (TBAD). However, surveillance computed tomography (CT) scans in post-TEVAR patients are associated with high radiation dose, thus resulting in potential risk of radiation-induced malignancy. In this study, we developed a patient-specific three-dimensional (3D) printed phantom with stent grafts in situ, then scanned the phantom with different CT protocols to determine the optimal scanning parameters for post-treatment patients. The CT scans were conducted with different $\mathrm{kVp}$ and pitch values $(80,100,120 \mathrm{kVp}$ and pitch of $1.2,1.5,2.0,2.5)$, resulting in a total of 12 datasets. Signal-to-noise ratio (SNR) was measured to determine and compare the image quality between different datasets. Results showed no significant differences in SNR between different $\mathrm{kVp}$ when the pitch value was 1.2. At low pitch values, a decrease in $\mathrm{kVp}$ from 120 to 80 led to a significant effective dose reduction by more than $20 \%$. SNR decreased by $30 \%$ when pitch was increased from 1.2 to 2.5 at $80 \mathrm{kVp}$, and $20 \%$ at $120 \mathrm{kVp}$. In contrast, there was only a 3.9\% decrease in SNR when $\mathrm{kVp}$ was reduced from 120 to 80 at pitch 1.2, and 15.9\% at pitch 2.5. High pitch with $100 \mathrm{kVp}$ can effectively reduce the dose while maintaining image quality.
\end{abstract}

Keywords: three-dimensional printing; type B aortic dissection; cardiovascular disease; endovascular aortic repair (EVAR); computed tomography angiography (CTA); dose reduction

\section{Introduction}

Thoracic endovascular aortic repair (TEVAR) can be a life-saving therapy for treating aortic disease. Compared to open surgical repair, TEVAR is a valid therapeutic option for type B aortic dissection (TBAD) because of its lower mortality, morbidity, and paraplegia rate in the past decade [1-3]. However, management algorithms remain controversial and long-term follow up is required to determine outcomes and reintervention rates [4-7].

Computed tomography angiography (CTA) is currently the preferred imaging modality for diagnosis, treatment planning, and follow up for AD patients. With improved spatial and temporal resolution available with modern computed tomography (CT) scanners, CTA plays an important role in the diagnosis and follow up of aortic dissection (AD) [8,9]. However, the high radiation dose associated with CTA is still a concern for the well-being of patients [10-12]. To monitor patients after TEVAR, the pursuit of high-resolution and 
higher-quality images may bring unnecessary radiation doses. Therefore, it is necessary to reduce the radiation dose while maintaining the quality of $\mathrm{CT}$ images.

Finding a balance between image quality and radiation risk has always been a challenging issue in achieving optimal scanning protocols. A number of studies describe different strategies to lower the radiation dose including the use of iterative reconstruction (IR) for reducing image noise, use of low tube kilovoltage peak $(\mathrm{kVp})$, and use of high-pitch protocols with fast speed CT scanners [13-15]. Despite these studies providing promising results, research is still limited with regard to the reduction of radiation dose for aortic dissection patients who require repeat scanning for many years.

Compared with preoperative planning when detail is paramount, postoperative patients could receive a lower dose for routine follow-up examinations to reduce cumulative radiation dose. Thus, the purpose of this study was to investigate the optimal CTA protocols for surveillance of TBAD patients after EVAR. We employed a patient-specific three-dimensional (3D) printed TBAD model with a stent-graft TEVAR in situ.

Patient-specific 3D printed phantoms have been proven to be valuable in multiple medical applications [16-21]. In our previous study, we described how we developed a patient-specific 3D printed TBAD aortic model, and confirmed its accuracy in resembling the mechanical and radiological properties of in vivo imaging under CT scanning modalities [22]. In this study, we extended our application of a 3D printed aorta model to focus on the study of optimal CT protocols by deploying a bespoke stent graft inside the true lumen of the $\mathrm{AD}$ aorta model to simulate the TEVAR process, and then scanning the model with different CT protocols.

\section{Materials and Methods}

Suitable patient data and materials were required for the preparation and implementation of the 3D printed aorta model used in the study. These components were identified and then utilized as per the methods outlined in the following sections.

\subsection{Selection of Sample Case and Segmentation}

Contrast-enhanced CT (CECT) image data of 11 patients who had TBAD were deidentified and retrieved from public hospital records between November 2015 and March 2016 after ethics approval (Curtin Human Research Ethics (HRE) Committee, approval number: HRE2018-0087). One of the patient datasets (CECT performed on a 128-slice CT with $120 \mathrm{kVp}$ tube potential, 128 reference $\mathrm{mAs}$, and $1.0 \mathrm{~mm}$ reconstructed slice thickness) was selected due to high image quality and contrast medium being present in the false lumen, allowing for identification of the aortic shape. The selected CT image dataset was imported into 3D Slicer (Version 4.9.0, www.slicer.org accessed on 26 July 2021; MA, USA) to proceed with segmentation. The chosen segment had a perfect description of both lumens and intimal flap and the border of the aortic wall could be clearly identified (Figure 1). After segmentation, a 3D outer surface reconstruction model was generated. Post-processing of the 3D reconstruction model was necessary to ensure model integrity and printing success including checking the thickness, filling of defects (for example, holes inside the models, discontinuous surfaces), and surface smoothing. The 3D reconstruction model was transferred into the stereolithography (STL) format for post cleaning and preprint checking. The final model was directly ordered from the online customer service of 3D Systems Inc. (Valencia, CA, USA). 


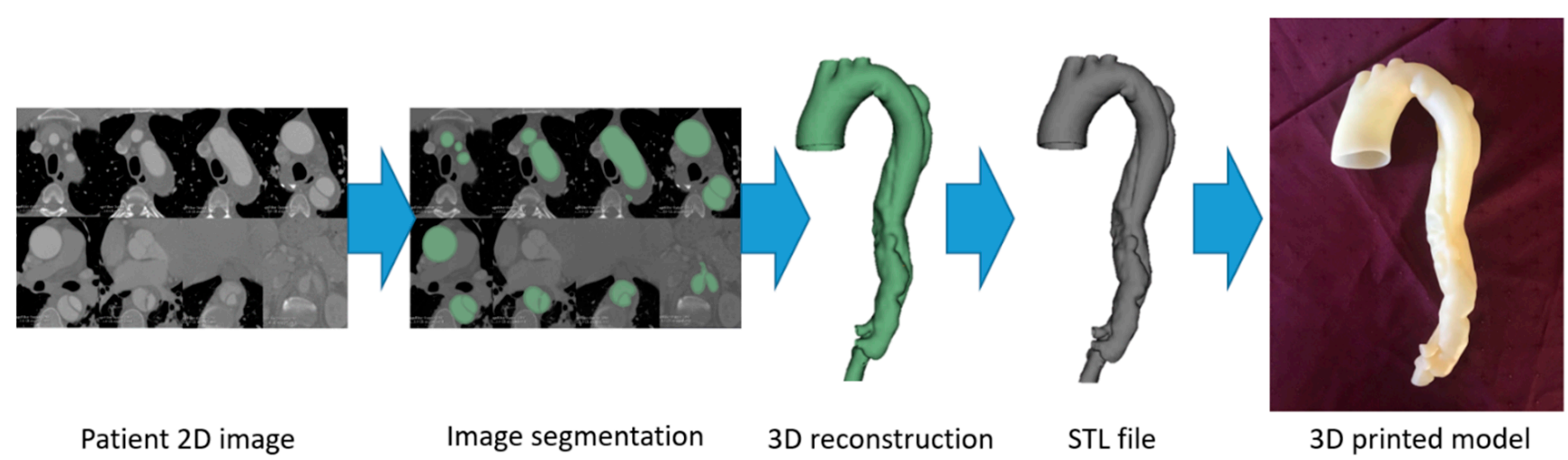

Figure 1. A 3D printed model was produced by the following five steps: image acquisition, image segmentation, surface mesh generation, stereolithographic (STL) post-processing, and printing.

\subsection{Selection of 3D Printing Materials}

The selection of a suitable printing material depends on three considerations: the mechanical properties, radiological properties, and transparency. To represent the mechanical properties of the human aorta, the material of the model must have similar flexibility and elasticity. The tensile strength in elderly patients with cardiovascular disease should be around $0.39 \mathrm{MPa}$ and have a modulus of elasticity of 0.628 [23]. The elongation at breaking point of the aorta is around $82 \%$ to $140 \%$ and the hardness is between Shore A12 and A18 throughout the ascending, descending, and abdominal aorta [24].

Based on our previous study, we chose four different 3D printing materials that had mechanical properties close to that of patients with aortic dissection [22]. In terms of transparency, a completely transparent model would enhance stent graft deployment without the need for imaging and contrast. This would enable good vision of intravascular structures and be useful in academic applications. However, a transparent elastomer printing material was not available from commercial companies, therefore, a translucent material was the next best option. After scanning samples of several available materials with the current clinical thoracic CTA protocol, one of the materials, Visijet CE-NT (3D systems Inc., Wilsonville, OR, USA), was selected as it had the same radiological properties to in vivo aorta [22]. The reason for choosing Visijet CE-NT is due to the following reasons: first, it is a rubber-like material suitable for printing aorta-like models. Second, it has a light yellow and translucent appearance allowing for visualization of the deployed stent grafts inside the aorta. Third, the Visijet CE-NT material has two different hardnesses, A30 and A70, with A30 having a tensile strength between 0.2 and 0.4 , which is close to elderly cardiovascular tissue properties. Finally, our recent study confirms that Visijet CE-NT A30 is the most appropriate material for printing aorta models because its $\mathrm{CT}$ attenuation is similar to that of the contrast-enhanced CT images of aortic dissection [22].

\subsection{Deployment of Stent Graft}

Three commercially available thoracic stent grafts (Medtronic) were deployed into the model after planning and sizing by an experienced vascular surgeon. The proximal piece deployed into the arch to cover the subclavian artery measured a length of $150 \mathrm{~mm}$ and diameter tapering from 42 to $38 \mathrm{~mm}$, which was placed in the aortic arch. The second overlapping piece was $90 \mathrm{~mm}$ long by $31 \mathrm{~mm}$ in diameter. The final distal piece measured $94 \mathrm{~mm}$ in length and $25 \mathrm{~mm}$ in diameter (Figure 2). 


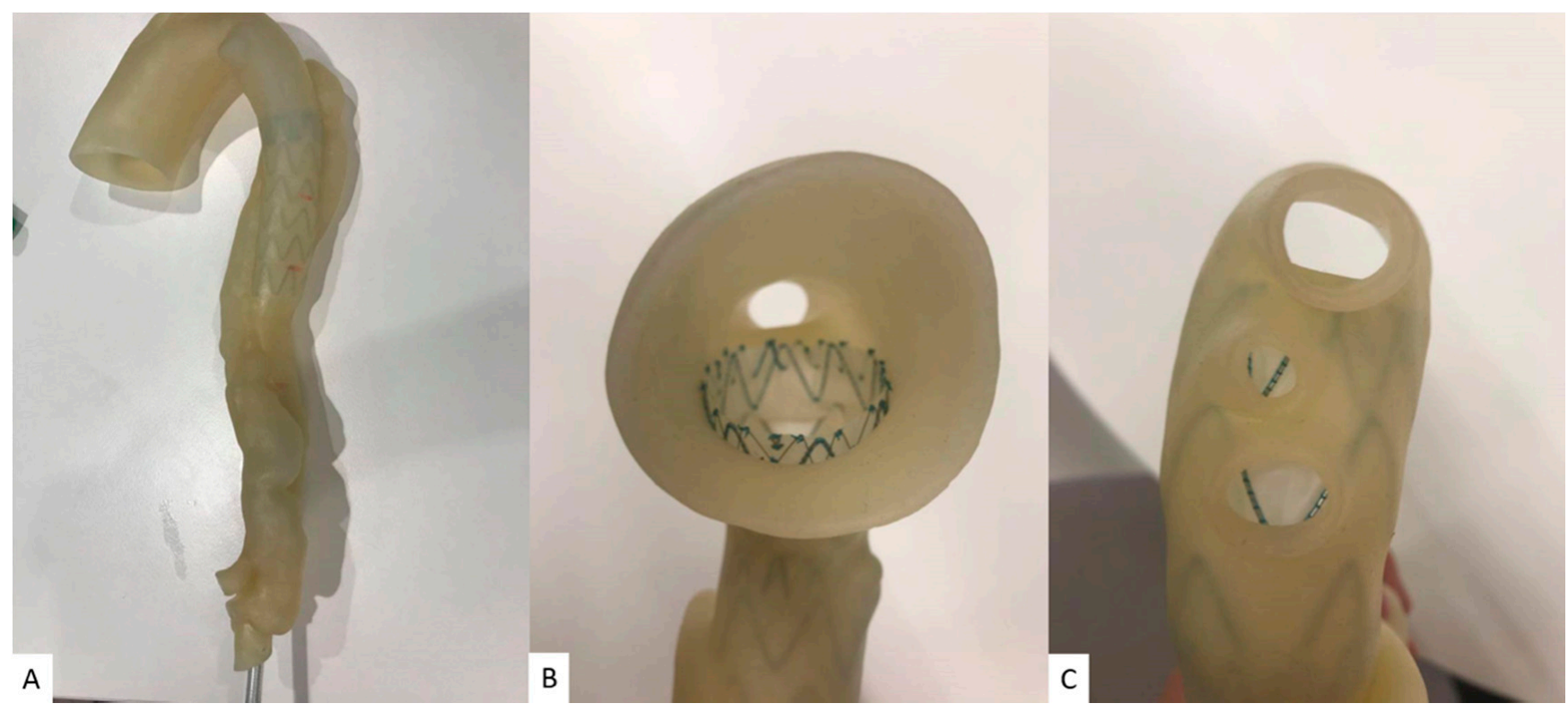

Figure 2. Stent graft deployed in the 3D printed model. (A) Deployed stent graft visible through model wall. (B) Axial view from proximal arch. (C) Caudal view down arch vessels.

\subsection{Aortic CTA Scanning Protocols}

The 3D printed TBAD model was placed in a plastic container that was filled with a contrast medium to simulate CECT examinations. Due to the flexibility of the model, air bubbles inside the model were expelled by aspiration via a syringe (Figure 3). The iohexol contrast medium OmnipaqueTM 350 (GE Healthcare Australia Pty Ltd., New South Wales, Australia), which is routinely used in contrast-enhanced CT scans, was diluted to $7 \%$ with a resulting CT attenuation of $300 \mathrm{HU}$, similar to that of routine CECT. The model was scanned by a 192-slice CT scanner (Siemens Force, Siemens Healthcare, Forchheim, Germany). The CECT scans were conducted with different $k V p$ and pitch values $(80,100$, $120 \mathrm{kVp}$ and pitch of $1.2,1.5,2.0,2.5)$, resulting in a total of 12 datasets. A slice thickness of $1.0 \mathrm{~mm}$ with a $0.5 \mathrm{~mm}$ reconstruction interval was applied to all images. An iterative reconstruction technique was used (advanced modelled iterative reconstruction (ADMIRE) algorithm with level 3, Siemens Healthcare) and a tissue convolution kernel of Bv40.

\subsection{Quantitative Assessment of Image Quality}

In order to determine the image quality in these CTA protocols, the image quality was quantitatively evaluated by measuring the signal-to-noise ratio (SNR) of the proximal and middle descending aorta. Regions of interest (ROI) were placed in the true and false lumens with an area of $25 \mathrm{~mm}^{2}$ (containing at least 300 voxels) to measure SNR. Figure 4 shows the ROI measurement of SNR in the aorta of the model. To minimize the difference between observers, the SNR measurement was repeated three times at each location, and the average value was used to minimize the difference between observers, and the average value between two observers was used as the final result.

\subsection{Radiation Dose}

The volume CT dose index (CTDI vol) and dose length product (DLP) were recorded and compared between different CTA scanning protocols. The effective dose was calculated using a tissue conversion coefficient of $0.014 \mathrm{mSv} / \mathrm{mGy} / \mathrm{cm}$, which is commonly used for the calculation of chest CT dose [25]. 




Figure 3. The CT scans were performed on the post stent graft deployed aorta model with different scanning protocols. (A) The model placed in a customized acrylic box for scanning. (B) The process of using the syringe to extract the internal air bubbles after pouring the contrast. (C) The final model ready to be scanned.
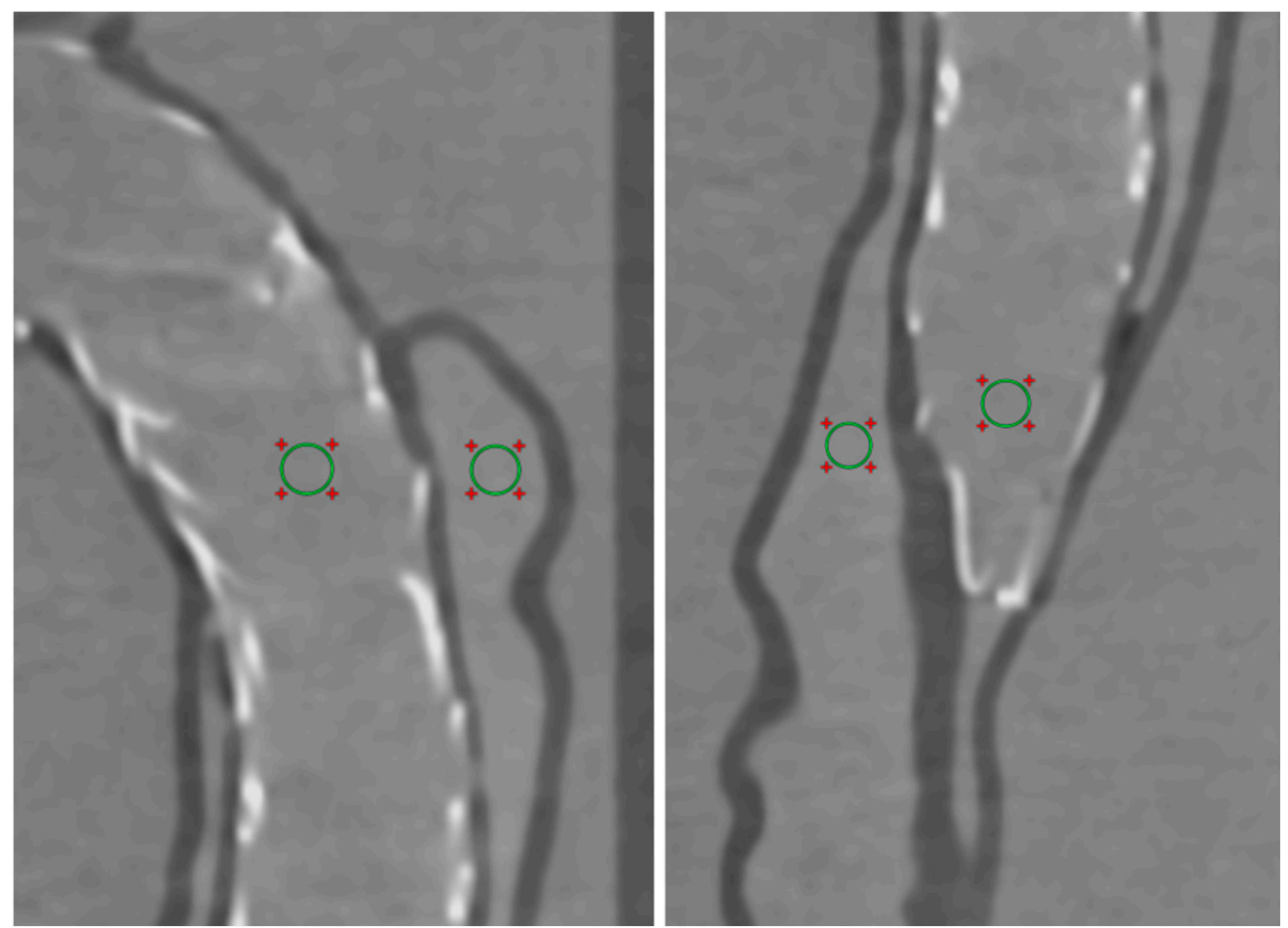

Figure 4. ROIs were placed inside the aortic model scan data to measure SNR. Four measuring points were used in each dataset including true and false lumen at proximal and middle descending aorta. ROIs: regions of interest, SNR: signal-to-noise ratio. 


\subsection{Statistical Analysis}

All data were imported into SPSS 26.0 (IBM Corporation, Armonk, NY, USA) for statistical analysis. A paired sample t-test was used to determine whether there were any significant differences in SNR measured with different CPA protocols. A P value of less than 0.05 indicated a statistically significant difference.

\section{Results}

Different CTA imaging protocols were successfully tested on the 3D printed model. All measurements were performed by two observers, with excellent correlation between the observers. Table 1 shows the SNR measurements at images acquired with different CTA protocols. SNR measurements did not vary significantly when the $k V p$ value range was close such as a comparison between 80 and $100 \mathrm{kVp}$ or between 100 and $120 \mathrm{kVp}(p>0.05)$, regardless of the pitch values. However, the measurements showed that at pitch 1.5, 2.0, and 2.5, SNR at $120 \mathrm{kVp}$ was significantly higher than $80 \mathrm{kVp}(p<0.05)$ in both true and false lumens. SNR showed no significant differences between different $\mathrm{kVp}$ when the pitch value was 1.2. At low pitch values such as 1.2 and 1.5, a decrease in $\mathrm{kVp}$ from 120 to 80 led to a significant effective dose reduction by more than $20 \%$.

Unlike $\mathrm{kVp}$, a variation of pitch values can significantly affect SNR measurements. When the $\mathrm{kVp}$ value is kept constant, SNR showed significant differences with different pitch values. SNR measured significantly higher in images acquired with low pitch values such as 1.2 and 1.5, compared to those acquired with the high 2.0 and 2.5 protocols $(p<0.05)$, regardless of the location of measurements. SNR measurements showed that image quality significantly dropped when the pitch value increased from 1.5 to 2.0 in all kinds of $\mathrm{kVp}$ protocols $(p<0.05)$. SNR dropped 30\% when pitch value was raised from 1.2 to 2.5 at $80 \mathrm{kVp}$, and $20 \%$ at $120 \mathrm{kVp}$. In contrast, SNR dropped only $3.9 \%$ when $\mathrm{kVp}$ was reduced from 120 to 80 at pitch 1.2 , and $15.9 \%$ at pitch 2.5 .

Under low pitch protocols such as 1.2 and 1.5, there were no significant differences in SNR measurements between the proximal and middle descending aorta. In contrast, at high pitch value protocols such as 2.0 and 2.5 , the proximal descending aorta tended to have a higher image quality than the middle descending aorta, especially in the false lumen. Figure 5 is an example showing the reformatted images of these CTA protocols. When $\mathrm{kVp}$ decreased to 80 , image noise was increased with the use of high pitch value protocols such as 2.0 and 2.5, as shown in Figure 5. However, the shape of the stent graft and the location of the intimal flap are clearly displayed in these images, despite the use of low-dose protocols. 
Table 1. SNR measurements at images acquired with different CTA protocols. SNR, signal-to-noise ratio; CTDIvol, volume CT dose index; DLP, dose length product.

\begin{tabular}{|c|c|c|c|c|c|c|c|c|c|c|c|c|}
\hline \multirow{2}{*}{$\begin{array}{c}\text { Tube Voltage } \\
\text { Pitch Value }\end{array}$} & \multicolumn{4}{|c|}{$80 \mathrm{kVp}$} & \multicolumn{4}{|c|}{$100 \mathrm{kVp}$} & \multicolumn{4}{|c|}{$120 \mathrm{kVp}$} \\
\hline & 1.2 & 1.5 & 2.0 & 2.5 & 1.2 & 1.5 & 2.0 & 2.5 & 1.2 & 1.5 & 2.0 & 2.5 \\
\hline $\begin{array}{l}\text { True lumen of proximal } \\
\text { descending aorta }\end{array}$ & $23.93 \pm 9.34$ & $20.30 \pm 1.69$ & $19.64 \pm 1.03$ & $13.70 \pm 1.42$ & $27.60 \pm 1.47$ & $22.19 \pm 2.14$ & $19.14 \pm 3.75$ & $12.60 \pm 0.38$ & $25.64 \pm 4.69$ & $25.10 \pm 2.74$ & $19.93 \pm 0.67$ & $19.23 \pm 3.74$ \\
\hline $\begin{array}{l}\text { True lumen of middle } \\
\text { descending aorta }\end{array}$ & $25.29 \pm 7.43$ & $25.10 \pm 3.58$ & $18.66 \pm 1.19$ & $16.62 \pm 1.28$ & $25.65 \pm 2.16$ & $25.91 \pm 0.29$ & $14.74 \pm 0.58$ & $18.40 \pm 1.92$ & $23.68 \pm 2.33$ & $26.21 \pm 0.26$ & $20.61 \pm 1.24$ & $19.81 \pm 1.04$ \\
\hline $\begin{array}{l}\text { False lumen of middle } \\
\text { descending aorta }\end{array}$ & $18.12 \pm 2.80$ & $22.13 \pm 1.54$ & $10.11 \pm 0.60$ & $12.47 \pm 0.54$ & $20.86 \pm 2.23$ & $26.27 \pm 3.77$ & $15.62 \pm 0.77$ & $12.16 \pm 1.16$ & $20.15 \pm 1.29$ & $29.91 \pm 4.84$ & $14.03 \pm 0.80$ & $13.53 \pm 0.51$ \\
\hline DLP (mGy/cm) & 2.8 & 2.9 & 2.2 & 2.1 & 3.9 & 3.8 & 2.7 & 2.4 & 4.1 & 3.8 & 2.8 & 2.5 \\
\hline Effective dose (mSv) & 0.04 & 0.04 & 0.03 & 0.03 & 0.05 & 0.05 & 0.04 & 0.03 & 0.06 & 0.05 & 0.04 & 0.04 \\
\hline
\end{tabular}




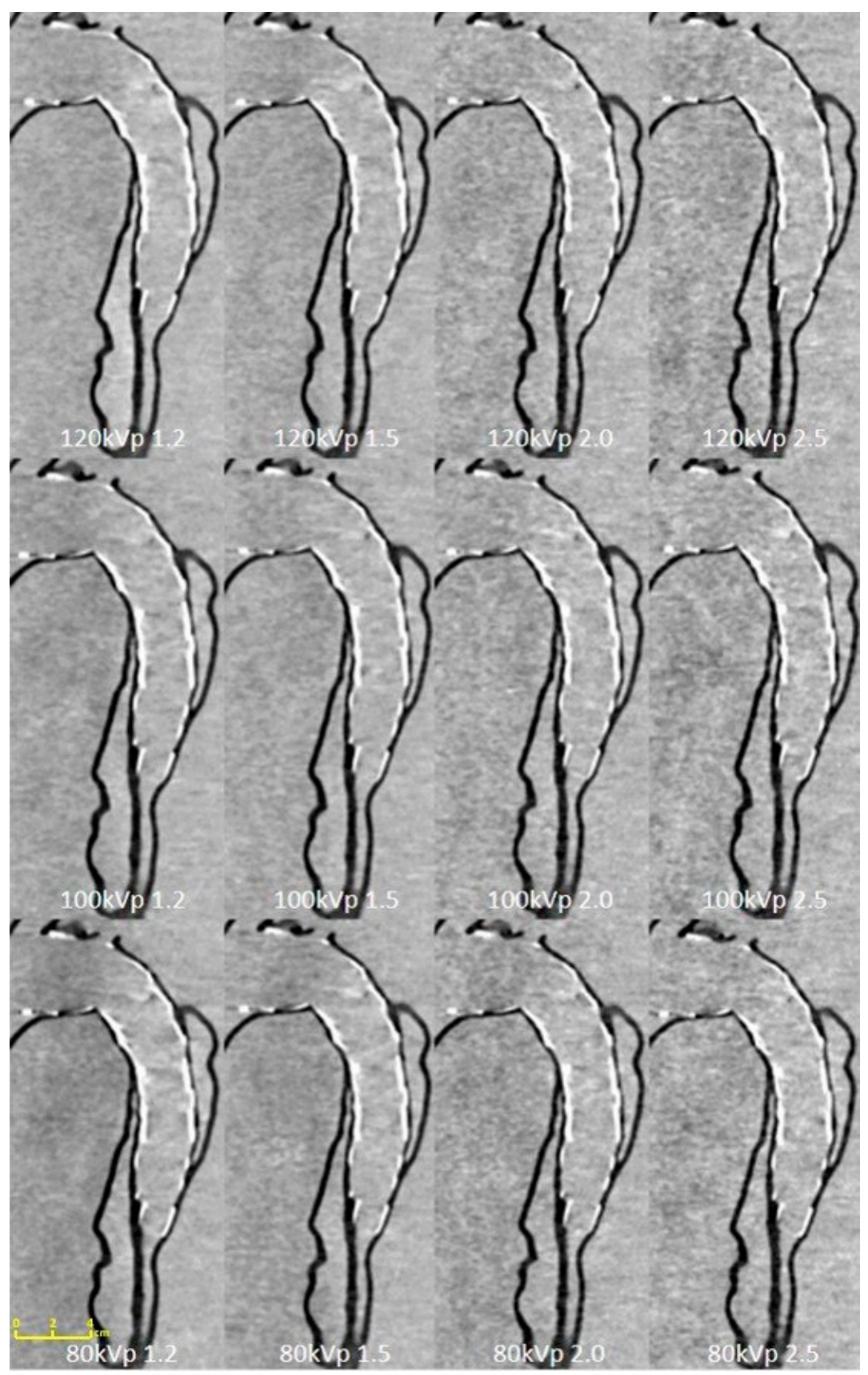

Figure 5. Sagittal reformatted images of the CTA protocols. When $\mathrm{kVp}$ decreased to 80 , image noise increased with the use of high pitch value protocols such as 2.0 and 2.5. CTA: computed tomography angiography, kVp: kilovoltage peak.

\section{Discussion}

In this study, we simulated a postprocedural surveillance CT scan of a patient with TBAD with different scanning protocols on a 3D printed aortic model. Quantitative assessment of image quality showed no significant differences between different $\mathrm{kVp}$ when the pitch value was 1.2 . More than $20 \%$ dose reduction was achieved with the use of low $\mathrm{kVp}$ such as 80 . Compared to the change in $\mathrm{kVp}$, image quality had significant differences between low and high pitch values, although there was no significant impact on the visualization of the stent graft and other structures. Thus, a low-dose aortic CTA is suggested for the follow-up of patients after EVAR.

For patients with aortic dissection, follow-up CT is mandatory and continues for many years $[8,11,26]$. Various dose-reduction strategies continue to develop with the 
advancement of CT imaging technology, striving to reduce the risk of patients being exposed to excessive radiation while maintaining image quality including low $\mathrm{kVp}$, use of IR algorithms, tube current modulation, high-pitch protocol, and use of dual energy CT $[10,15,25]$. Low-dose CTA using low $\mathrm{kVp}$ and high-pitch protocols has been proven to achieve diagnostic image quality compared to the standard aortic CTA protocol while significantly reducing radiation dose $[10,13,27]$. However, the image quality research for post TEVAR follow-up patients is still limited [28,29]. This study adds valuable information to the current literature by exploring a variety of CTA protocols and supporting the value of patient-specific 3D printed phantoms.

A CT protocol with high-pitch is available with fast speed CT scanners and will decrease radiation dose significantly when the pitch is increased. However, pitch value also significantly affects image quality with compromising spatial resolution; higher pitch leads to an increase in image noise, which could possibly affect the diagnostic quality. This has been confirmed by our study as image noise was significantly raised when the pitch was increased (Figure 5). Despite this potential limitation, some other studies have still shown the possibility of using high-pitch CTA protocols in the diagnosis of aortic dissection without losing image quality [10]. Shen et al. claimed that the use of a proper iterative reconstruction algorithm is able to maintain image quality while reducing the dose by using a low $\mathrm{kVp}$, high pitch scanning protocol [13]. Freyhardt et al. also investigated low-kV CT protocols with IR, allowing for CTA of the entire aorta with excellent image quality and diagnostic confidence with a dose reduction of up to $80 \%$ compared to $120 \mathrm{kV}$ [27]. In our study, we showed the influence of pitch value on image quality, which is more obvious than adjusting $\mathrm{kVp}$, especially at low $\mathrm{kVp}$.

The novelty of this study lies in the use of a personalized 3D printed aortic model to simulate TEVAR of TBAD. Use of 3D printed models to study optimal CT scanning protocols has been supported in previous studies including the assessment of coronary artery plaques [30], coronary stenting [31] and detection of pulmonary embolism [32,33]. In order to evaluate the impact of different protocols on radiation dose and image quality, $\mathrm{CT}$ scans have to be conducted multiple times with different scanning parameters. However, it would be unethical to scan the same patient multiple times and performing this experiment with different patients would lose accuracy due to different body types. With the development of 3D printing technology and the advancement of its application in the medical field, patient-specific 3D printed models could potentially become the new option for these kinds of experiment $[16,18,34]$.

Previous literature has discussed the use of 3D printed models to plan stent graft treatment in patients. Tong et al. deployed stent grafts in short 3D printed aortic models with thoracoabdominal aortic disease. They used these models to guide pre-fenestration of the stent grafts before re-deploying them in patients, believing that 3D printing technology could potentially improve both the accuracy and efficiency of the TEVAR procedure [35]. $\mathrm{Li}$ et al. also used 3D printed models to guide total endovascular repair for a patient who had a stent graft incorrectly deployed in the false lumen [36]. Zhang et al. performed prefenestration on a stent graft in a 3D printed model. They thought stent graft pre-fenestration guided by the 3D printed aortic model provided a new solution for aortic dissection treatment planning and claimed that this could improve accuracy of the fenestration location [37]. Although these previous studies of stent graft deployment inside 3D models are of interest, most did not specify the materials they used to make the models, or attempted to use materials that have mechanical and radiological properties close to the real human aorta. In our study, we provide a new possibility for the medical application of $3 \mathrm{D}$ printing by using $3 \mathrm{D}$ printing materials with accuracy in vivo representation to obtain more realistic experimental data.

We recognize that there are some limitations to our experiment. First, despite a realistic 3D printed model being used for studying different CTA protocols, the model was not placed in an environment that simulated normal surrounding anatomic regions such as the lungs, ribs, spinal column, or heart. Therefore, the radiation dose associated with these 
protocols were lower than the actual values reported by other studies. In addition, the $3 \mathrm{D}$ printed aorta model was placed in a customized box and immersed inside a dilute contrast medium instead of the perfusion of contrast medium into the lumen. Furthermore, the contrast was not flowing. Future research could focus on improving this aspect of the model. Connecting the 3D printed aortic model to a pulsatile flow circuit with a cardiac pump or printing a controllable syringe pump could be a solution as this will allow for accurate measurement under more dynamic conditions. This can be addressed by further studies when our printing capabilities meet these requirements. Despite this limitation, the current measurements are still acceptable and consistent with what has been reported in the literature regarding measurements of dimensional accuracy based on 3D printed static cardiovascular models [21]. Second, no contrast-to-noise ratio (CNR) was measured. In this study, only SNR was measured to determine image quality because the model was immersed in the contrast medium. A more robust conclusion could be reached with the measurement of both SNR and CNR when the 3D printed models are placed in a realistic chest phantom in future studies. Third, the measurement of SNR and effective dose could be different in patients. Although we selected a 3D printing material that had mechanical and radiological properties approximating those in the human, due to the limited choice of commercial 3D printing materials, we could not exactly match the human aorta.

Different slice thicknesses, use of IR strengths, and image post-processing algorithms to suppress the artifacts from stents could also affect the SNR measurements. Additionally, we did not undertake a qualitative assessment of image quality in this study. In future research, the application value of $3 \mathrm{D}$ printed models could be verified through scoring by clinical imaging professionals.

\section{Conclusions}

In this study, we demonstrated the use of a 3D printed full-sized aortic dissection model to investigate the possibility of lower radiation dose for the surveillance of TBAD post TEVAR using CT with different scanning parameters. Low-dose CT with acceptable image quality can be achieved by lowering the tube voltage. Compared to adjusting $\mathrm{kVp}$, increasing the pitch can effectively reduce the radiation dose, but will also reduce the overall image quality. High pitch with medium $\mathrm{kVp}$ can effectively reduce the dose while maintaining image quality. The contribution of 3D printing to medical applications was also explored in this study. However, since the model was not placed in a realistic chest cavity environment, the radiation dose reports in this study were lower than the actual values. Improvements in materials and design could add further details and will be explored in future studies.

Author Contributions: Conceptualization, C.-A.W., A.S., and Z.S.; Methodology, C.-A.W., A.S., and S.J.; Formal analysis, C.-A.W.; Investigation, C.-A.W., A.S., S.J., and Z.S.; Data curation, C.-A.W. and Z.S.; Writing-original draft preparation, C.-A.W.; Writing-review and editing, all authors; Visualization, C.-A.W.; Supervision, A.S., S.J., and Z.S.; Project administration, Z.S. All authors have read and agreed to the published version of the manuscript.

Funding: This research received no external funding.

Institutional Review Board Statement: The study was conducted according to the guidelines of the Declaration of Helsinki, and approved by the Human Research Ethics Committee of CURTIN UNIVERSITY (approval number: HRE2018-0087).

Informed Consent Statement: Patient consent was waived due to the use of anonymized data for 3D printing purposes with no patient information identified.

Data Availability Statement: The datasets used during the current study are not publicly available due to strict requirements set out by the Human Ethics Research Committee regarding the storage and use of the data by authorised investigators.

Acknowledgments: The authors would like to thank Tom Tiang from the Perth Children's Hospital for his assistance in CT scanning the 3D printed models. 
Conflicts of Interest: The authors declare no conflict of interest.

\section{References}

1. Szeto, W.Y.; McGarvey, M.; Pochettino, A.; Moser, W.; Hoboken, A.; Cornelius, K.; Woo, E.Y.; Carpenter, J.P.; Fairman, R.M.; Bavaria, J.E. Results of a new surgical paradigm: Endovascular repair for acute complicated type B aortic dissection. Ann. Thorac. Surg. 2008, 86, 87-94. [CrossRef]

2. Thrumurthy, S.G.; Karthikesalingam, A.; Patterson, B.O.; Hinchliffe, R.J.; Loftus, I.M.; Thompson, M.M. A systematic review of mid-term outcomes of thoracic endovascular repair (TEVAR) of chronic type B aortic dissection. Eur. J. Vasc. Endovasc. Surg. 2011, 42, 632-647. [CrossRef] [PubMed]

3. Bavaria, J.E.; Brinkman, W.T.; Hughes, C.; Khoynezhad, A.; Szeto, W.Y.; Azizzadeh, A.; Lee, W.A.; White, R.A. Outcomes of thoracic endovascular aortic repair in acute type B aortic dissection: Results from the Valiant United States investigational device exemption study. Ann. Thorac. Surg. 2015, 100, 802-809. [CrossRef]

4. Nienaber, C.A.; Kische, S.; Rousseau, H.; Eggebrecht, H.; Rehders, T.C.; Kundt, G.; Glass, A.; Scheinert, D.; Czerny, M.; Kleinfeldt, T.; et al. Endovascular repair of type B aortic dissection: Long-term results of the randomized investigation of stent grafts in aortic dissection trial. Circ. Cardiovasc. Interv. 2013, 6, 407-416. [CrossRef]

5. Huang, C.-Y.; Hsu, H.-L.; Chen, P.-L.; Chen, I.-M.; Hsu, C.-P.; Shih, C.-C. The impact of distal stent graft-induced new entry on aortic remodeling of chronic type B dissection. Ann. Thorac. Surg. 2018, 105, 785-793. [CrossRef]

6. Hossack, M.; Patel, S.; Gambardella, I.; Neequaye, S.; Antoniou, G.A.; Torella, F. Endovascular vs. medical management for uncomplicated acute and sub-acute type B aortic dissection: A meta-analysis. Eur. J. Vasc. Endovasc. Surg. 2020, 59, 794-807. [CrossRef] [PubMed]

7. Burke, C.R.; Bavaria, J.E. The role of thoracic endovascular repair in chronic type B aortic dissection. Semin. Thorac. Cardiovasc. Surg. 2020, 32, 21-24. [CrossRef]

8. Pape, L.A.; Awais, M.; Woznicki, E.M.; Suzuki, T.; Trimarchi, S.; Evangelista, A.; Myrmel, T.; Larsen, M.; Harris, K.M.; Greason, K.; et al. Presentation, diagnosis, and outcomes of acute aortic dissection: 17-year trends from the international registry of acute aortic dissection. J. Am. Coll. Cardiol. 2015, 66, 350-358. [CrossRef]

9. Fukui, T. Management of acute aortic dissection and thoracic aortic rupture. J. Intensive Care. 2018, 6, 1-8. [CrossRef] [PubMed]

10. Apfaltrer, P.; Hanna, E.L.; Schoepf, U.J.; Spears, J.R.; Schoenberg, S.O.; Fink, C.; Vliegenthart, R. Radiation dose and image quality at high-pitch CT angiography of the aorta: Intraindividual and interindividual comparisons with conventional CT angiography. Am. J. Roentgenol. 2012, 199, 1402-1409. [CrossRef]

11. Sun, Z.; Al Moudi, M.; Cao, Y. CT angiography in the diagnosis of cardiovascular disease: A transformation in cardiovascular CT practice. Quant. Imaging Med. Surg. 2014, 4, 376-396.

12. Hoffmann, A.; Engelfriet, P.; Mulder, B. Radiation exposure during follow-up of adults with congenital heart disease. Int. J. Cardiol. 2007, 118, 151-153. [CrossRef] [PubMed]

13. Shen, Y.; Sun, Z.; Xu, L.; Li, Y.; Zhang, N.; Yan, Z.; Fan, Z. High-pitch, low-voltage and low-iodine-concentration CT angiography of aorta: Assessment of image quality and radiation dose with iterative reconstruction. PLoS ONE 2015, 10, e0117469. [CrossRef] [PubMed]

14. Felmly, L.M.; Cecco, C.N.; Schoepf, U.J.; Varga-Szemes, A.; Mangold, S.; McQuiston, A.D.; Litwin, S.E.; Bayer II, R.R.; Vogl, T.J.; Wichmann, J.L. Low contrast medium-volume third-generation dual-source computed tomography angiography for transcatheter aortic valve replacement planning. Eur. Radiol. 2017, 27, 1944-1953. [CrossRef] [PubMed]

15. Euler, A.; Taslimi, T.; Eberhard, M.; Kobe, A.; Reeve, K.; Zimmermann, A.; Krauss, A.; Gutjahr, R.; Schmidt, B.; Alkadhi, H. Computed tomography angiography of the aorta-Optimization of automatic tube voltage selection settings to reduce radiation dose or contrast medium in a prospective randomized rrial. Investig. Radiol. 2021, 56, 283-291. [CrossRef] [PubMed]

16. Sun, Z. Insights into 3D printing in medical applications. Quant. Imaging Med. Surg. 2019, 9, 1-5. [CrossRef]

17. Byrne, N.; Belasco, M.; Tandon, A.; Hussain, T. A systematic review of image segmentation methodology, used in the additive manufacture of patient-specific 3D printed models of the cardiovascular system. JRSM Cardiovasc. Dis. 2016, 5, 2048004016645467. [CrossRef]

18. Foley, T.A.; El Sabbagh, A.; Anavekar, N.S.; Williamson, E.E.; Matsumoto, J.M. 3D-Printing: Applications in Cardiovascular Imaging. Curr. Radiol. Rep. 2017, 5, 1-13. [CrossRef]

19. Giannopoulos, A.A.; Steigner, M.; George, E.; Barile, M.; Hunsaker, A.R.; Rybicki, F.J.; Mitsouras, D. Cardiothoracic applications of 3-D printing. J. Thorac. Imaging 2016, 31, 253-272. [CrossRef]

20. Eshkalak, S.K.; Ghomi, E.R.; Dai, Y.; Choudhury, D.; Ramakrishna, S. The role of three-dimensional printing in healthcare and medicine. Mater. Des. 2020, 108940. [CrossRef]

21. Sun, Z. Clinical applications of patient-specific 3D printed models in aardiovascular disease: Current status and future directions. Biomolecules 2020, 10, 1577. [CrossRef] [PubMed]

22. Wu, C.-A.; Squelch, A.; Sun, Z. Investigation of three-dimensional printing materials for printing aorta model replicating Type B aortic dissection. Curr. Med. Imaging 2021, 17, 843-849.

23. Ratinam, R.; Quayle, M.; Crock, J.; Lazarus, M.; Fogg, Q.; McMenamin, P. Challenges in creating dissectible anatomical 3D prints for surgical teaching. J. Anat. 2019, 234, 419-437. [CrossRef] 
24. Riedle, H.; Mukai, B.; Molz, P.; Franke, J. Determination of the mechanical properties of aortic tissue for 3D printed surgical models. In Proceedings of the 2018 11th Biomedical Engineering International Conference (BMEiCON), Chiang Mai, Thailand, 21-24 November 2018; pp. 1-5. [CrossRef]

25. McCollough, C.H.; Primak, A.N.; Braun, N.; Kofler, J.; Yu, L.; Christner, J. Strategies for reducing radiation dose in CT. Radiol. Clin. North Am. 2009, 47, 27-40. [CrossRef]

26. Tanaka, M.; Kimura, N.; Yamaguchi, A.; Adachi, H. In-hospital and long-term results of surgery for acute type A aortic dissection: 243 consecutive patients. Ann. Thorac. Cardiovasc. Surg. 2012, 18, 18-23. [CrossRef]

27. Freyhardt, P.; Solowjowa, N.; Böning, G.; Kahn, J.; Aufmesser, B.; Haage, P.; Streitparth, F. CT-angiography of the aorta in patients with Marfan disease-High-pitch MDCT at different levels of tube voltage combined with Sinogram Affirmed Iterative Reconstruction. Clin. Imaging. 2018, 51, 123-132. [CrossRef]

28. Flors, L.; Leiva-Salinas, C.; Norton, P.T.; Patrie, J.T.; Hagspiel, K.D. Imaging follow-up of endovascular repair of type B aortic dissection with dual-source, dual-energy CT and late delayed-phase scans. J. Vasc. Interv. Radiol. 2014, 25, 435-442. [CrossRef] [PubMed]

29. Karmonik, C.; Duran, C.; Shah, D.J.; Anaya-Ayala, J.E.; Davies, M.G.; Lumsden, A.B.; Bismuth, J. Preliminary findings in quantification of changes in septal motion during follow-up of type B aortic dissections. J. Vasc. Surg. 2012, 55, 1419-1426. [CrossRef] [PubMed]

30. Sun, Z.; Ng, C.K.; Squelch, A. Synchrotron radiation computed tomography assessment of calcified plaques and coronary stenosis with different slice thicknesses and beam energies on 3D printed coronary models. Quant. Imaging Med. Surg. 2019, 9, 6-22. [CrossRef] [PubMed]

31. Sun, Z.; Jansen, S. Personalized 3D printed coronary models in coronary stenting. Quant. Imaging Med. Surg. 2019, 9, 1356-1367. [CrossRef]

32. Aldosari, S.; Jansen, S.; Sun, Z. Patient-specific 3D printed pulmonary artery model with simulation of peripheral pulmonary embolism for developing optimal computed tomography pulmonary angiography protocols. Quant. Imaging Med. Surg. 2019, 9, 75-85. [CrossRef] [PubMed]

33. Aldosari, S.; Jansen, S.; Sun, Z. Optimization of computed tomography pulmonary angiography protocols using 3D printed model with simulation of pulmonary embolism. Quant. Imaging Med. Surg. 2019, 9, 53-62. [CrossRef] [PubMed]

34. Kim, G.B.; Lee, S.; Kim, H.; Yang, D.H.; Kim, Y.-H.; Kyung, Y.S.; Kim, C.-S.; Choi, S.H.; Kim, B.J.; Ha, H.; et al. Three-dimensional printing: Basic principles and applications in medicine and radiology. Korean J. Radiol. 2016, 17, 182-197. [CrossRef] [PubMed]

35. Tong, Y.-H.; Yu, T.; Zhou, M.-J.; Liu, C.; Zhou, M.; Jiang, Q.; Liu, C.-J.; Li, X.-Q.; Liu, Z. Use of 3D printing to guide creation of fenestrations in physician-modified stent-grafts for treatment of thoracoabdominal aortic disease. J. Endovasc. Ther. 2020, 27, 385-393. [CrossRef] [PubMed]

36. Li, X.-R.; Tong, Y.H.; Li, X.Q.; Liu, C.J.; Liu, C.; Liu, Z. Total endovascular repair of an intraoperative stent-graft deployed in the false lumen of Stanford type A aortic dissection: A case report. World J. Clin. Cases 2020, 8, 954. [CrossRef]

37. Zhang, M.; Tong, Y.H.; Liu, C.; Li, X.Q.; Liu, C.J.; Liu, Z. Treatment of Stanford type A aortic dissection with triple pre-fenestration, reduced diameter, and three-dimensional-printing techniques: A case report. World J. Clin. Cases 2021, 9, 183. [CrossRef] 